\title{
Evaluating the Utility of the Pixel Value Ratio in the Determination of Time to Full Weight-bearing in Patients Undergoing Intramedullary Limb Lengthening
}

\author{
Anirejuoritse Bafor ${ }^{1}$, Molly E Duncan ${ }^{2}$, Christopher A lobst ${ }^{3}$
}

\begin{abstract}
Introduction: Limb lengthening using intramedullary nails is an increasingly popular method of achieving limb length equalisation. Currently, the decision regarding when to commence full weight-bearing (FWB) remains largely subjective. Objective criteria for determining the proper timing of FWB would be helpful to limb lengthening surgeons. This study examines using the pixel value ratio (PVR) as an objective method to determine the time to FWB for patients being lengthened with an intramedullary nail.

Materials and methods: A retrospective chart review of 42 patients who underwent unilateral lengthening of the femur was undertaken. The PVR of all four cortices of the regenerate bone was monitored throughout the distraction and consolidation stages to determine the ratio at the time of FWB.

Results: Clinically and radiologically determined FWB was achieved at a mean time of $125.7 \pm 30.1$ days from surgery. The mean PVR at the time of FWB was 0.94. The medial cortex healed fastest with a mean PVR of 0.96, while the posterior cortex healed slowest with a mean PVR of 0.92 . Conclusion: The PVR is a quick and reliable method to objectively assess the state of healing of the regenerate bone during distraction osteogenesis. We observed that there were no adverse effects when subjects commenced FWB when three out of the four cortices had a PVR of at least 0.93 .

Keywords: Distraction osteogenesis, Intramedullary limb lengthening, Pixel value ratio.

Strategies in Trauma and Limb Reconstruction (2020): 10.5005/jp-journals-10080-1461
\end{abstract}

\section{INTRODUCTION}

Limb lengthening is a procedure that has been performed for more than a century. 'The concept of distraction osteogenesis, originally described by llizarov, advanced the understanding of the biology involved in limb lengthening. ${ }^{2}$ This concept, combined with his stable external fixator device, allowed patients to achieve more reliable lengthening results compared to the previous methods. Recently, limb lengthening using an intramedullary lengthening nail has become popular. This technique has been shown to be accurate, consistent and avoids the pin site issues related to lengthening with an external fixator. ${ }^{3-5}$ Although the intramedullary device can help to protect the regenerate bone, the decisions regarding when to begin weight-bearing and how much weight to allow are still critical. Device failure can occur if too much weight is placed on the limb too early. Deciding the timing for allowing full ambulation is still largely a subjective process based on the quality and quantity of regenerate bone on the radiographs and the clinical status of the patient. ${ }^{6}$ Currently, most surgeons will allow a patient to advance to full weight-bearing (FWB) if the patient is already comfortable with partial weight-bearing, has appropriate range of motion (ROM) and the regenerate appears consolidated on at least three of the four cortices visible on the anteroposterior and lateral radiographs. A more objective tool to assist determining while FWB is safe to commence would be valuable for limb lengthening surgeons.

Dual-energy X-ray absorptiometry (DEXA) and quantitative computed tomography (QCT) scans have been proposed as noninvasive methods of monitoring bone healing. ${ }^{7}$ Each technique provides an objective assessment of the quantification of $\overline{1_{1,2} \text { Department of Orthopedic Surgery, Nationwide Children's Hospital, }}$ Columbus, Ohio, USA

Corresponding Author: Christopher A lobst, Department of Orthopedic Surgery, Nationwide Children's Hospital, Ohio, USA, Phone: +1 (614) 722-5175, e-mail: Christopher.lobst@nationwidechildrens.org How to cite this article: Bafor A, Duncan ME, lobst CA. Evaluating the Utility of the Pixel Value Ratio in the Determination of Time to Full Weight-bearing in Patients Undergoing Intramedullary Limb Lengthening. Strategies Trauma Limb Reconstr 2020;15(2):74-78.

Source of support: Nil

Conflict of interest: None

mineralisation of bone. For example, Saran and Hamdy used DEXA scans to monitor regenerate mineralisation and bone healing in $28 \mathrm{limb}$ lengthening procedures. They found a $3.6 \%$ fracture rate following removal of hardware. ${ }^{8}$ These tests, however, are expensive and not always routinely available. In addition, waiting on the test results delays the decision-making process, which makes routine clinical use impractical.

The pixel value ratio (PVR) can be determined from the digital $X$-rays obtained in the clinic. It has been shown to have a good correlation with DEXA scanning in determining the bone mineral density (BMD) of regenerate bone as a quantitative measure of mineralisation and healing during distraction osteogenesis with external fixators. ${ }^{9,10}$ The PVR can be calculated in the clinic setting and does not require any additional expense or radiation for the patient. This makes it a potentially attractive method to objectively measure the status of regenerate bone healing. The aim of this study 
was to determine if the PVR could be used to make a correlation between the amount of mineralisation of regenerate bone and the timing of FWB in patients undergoing intramedullary limb lengthening.

\section{Materials and Methods}

Following IRB approval from our institution, a retrospective analysis of data collected from 42 patients who underwent unilateral lengthening of the femur for limb length discrepancy (LLD) using the PRECICE nail at our facility between May 2017 and December 2019 was performed. There were 25 cases who had a congenital aetiology, 6 cases of posttraumatic growth arrest, 4 cases with a developmental aetiology, 4 cases resulting from infection, 2 cases following neoplastic conditions and 1 case from a metabolic cause of LLD. The mean age of the patients was $15.4 \pm 4$ years with a range of 9 to 31 years. There were 18 male and 24 female patients. The mean LLD was $4.2 \pm 2.1 \mathrm{~cm}$ with a range of 1.5 to $11 \mathrm{~cm}$. There were 19 right and 23 left limbs. Follow-up ranged from 4 to 35 months following surgery. All patients were FWB at the time of assessment.

All surgeries were carried out by the senior author. Distraction commenced 6-7 days following surgery at a rate of $0.75 \mathrm{~mm}$ per day. Patients were reviewed clinically at weekly intervals during the distraction phase of treatment and then every 3-4 weeks during the consolidation phase of treatment. The decision to commence FWB was based on the judgement of the senior author CAI, upon review of standard digital radiographs of the patients as well as their clinical status. Clinically, the patient must demonstrate painless, partial weight-bearing with a minimum knee ROM of 0 to 90 degrees. Radiographically, the regenerate was considered healed if bridging callus (i.e., a continuous column of bone) was visible on three out of the four cortices.

The PVR of the lateral, medial, anterior and posterior regions of the regenerate bone was assessed for each patient, from standard anteroposterior and lateral view digital radiographs obtained during regular follow-up visits using the "region of interest" program in GE Centricity picture archiving and communication systems (PACS) version 4.0.11E software (Fig. 1). The anterior and posterior PVRs were determined from lateral view images, while the medial and lateral ratios were determined from anteroposterior view images. The PVR is determined by dividing the average of the pixel values for the adjacent proximal and distal bone segments by the pixel value of the regenerate bone segment: ${ }^{10}$

0.5 (proximal segment pixel value + distal segment pixel value) regenerate bone segment pixel value

The raw pixel value decreases as adiopacity increases, so in the final expression of the PVR, an inverse ratio was used in order to present an increasing PVR as healing progresses. A ratio of 1 connotes similar density between regenerate bone and the adjacent normal bone. The radiographs were assessed retrospectively by two authors ( $A B$ and $M D$ ). Care was taken to ensure that hardware was not included while selecting the region of interest for analysis on the radiographs. All patients who had soft tissue overlap obscuring the region of interest on their radiographs were excluded from the study.

\section{Data Analysis}

The mean as a measure of central tendency was calculated where required using excel statistical package with variance expressed
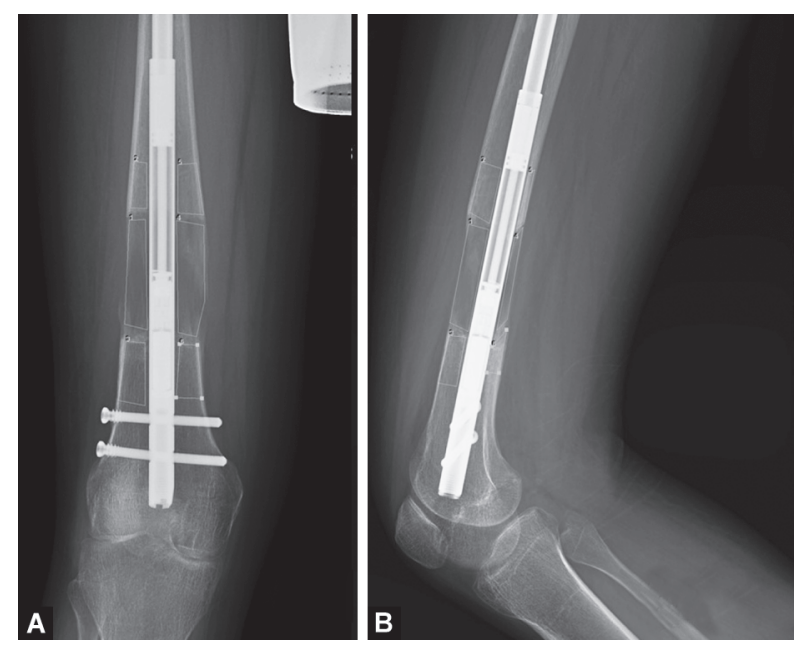

Figs $1 \mathrm{~A}$ and $\mathrm{B}$ : Digital radiographs of the distal femur showing measurement of the pixel densities for the regenerate as well as the adjacent proximal and distal cortices using the "region of interest" program in picture archiving and communication systems. (A) Anteroposterior view; (B) Lateral view

Table 1: Mean pixel value ratio for the various cortices at time of commencement of full weight-bearing

\begin{tabular}{ll}
\hline Cortex & Mean PVR \\
\hline Lateral & 0.93 \\
Medial & 0.96 \\
Anterior & 0.93 \\
Posterior & 0.92 \\
All cortices & 0.94 \\
\hline
\end{tabular}

PVR, pixel value ratio

as standard deviation. Scatter plots were designed to represent the trend of change of PVR over time. The mean PVR at the time of commencement of FWB was determined from the trendline of the charts for each cortex.

\section{Results}

The mean length obtained was $3.6 \pm 1.2 \mathrm{~cm}$ with a range of 2-6.2 cm. The mean time taken to achieve FWB was $125.7 \pm 30.1$ days with a range of 60-199 days. The mean PVR at the time of commencement of FWB was $0.93,0.96,0.93$ and 0.92 for the lateral, medial, anterior and posterior cortices, respectively, with an overall mean PVR of 0.94 (Table 1).

The medial cortex had a PVR value closest to 1 at the time of commencement of FWB, while the posterior cortex had the PVR value farthest from 1 (Table 1). The rate of change of the PVR is demonstrated in the scatter plots (Fig. 2).

\section{Discussion}

Deciding when to allow the patient to begin FWB remains a challenge in intramedullary limb lengthening. There is currently no purely objective manner with which to make this determination in the clinic. Most commonly, a subjective decision based on a combination of clinical and radiographic findings is used to decide when it may be safe to commence FWB. For limb lengthening using external fixators, visualisation of bridging callus, at least $2 \mathrm{~mm}$ 

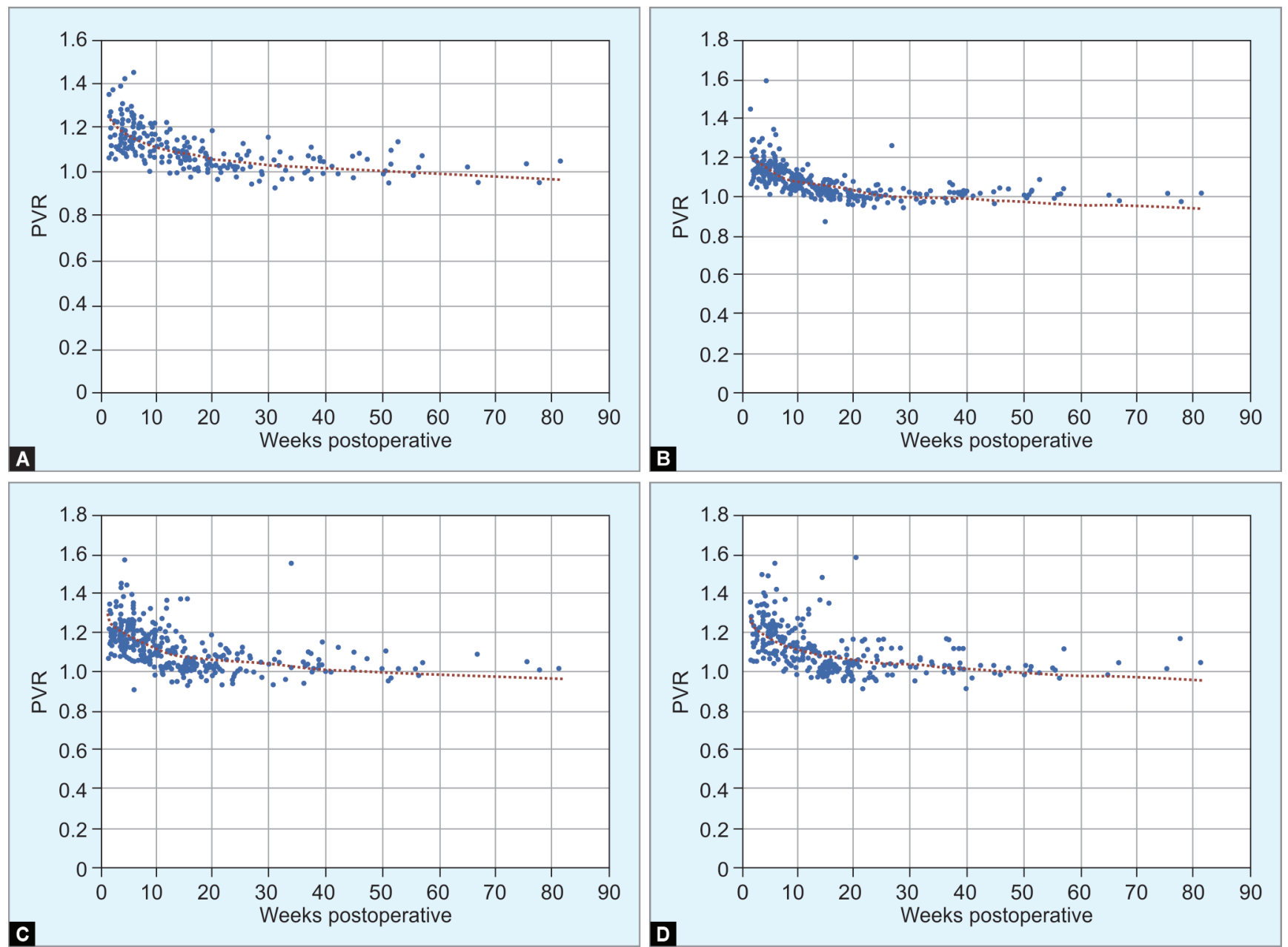

Figs 2A to D: Scatter plots with trendlines showing change of pixel value ratio (PVR) over time: (A) Lateral cortex; (B) Medial cortex; (C) Anterior cortex; (D) Posterior cortex

thick, on three out of the four cortices on plain anteroposterior and lateral radiographs has traditionally been considered the accepted radiographic criteria for removal. ${ }^{11,12}$ However, these measurements are a subjective process as evidenced by the high intra- and interobserver variability. 6,13 There are currently no established similar radiographic criteria for intramedullary lengthening patients.

Attempts to characterise regenerate bone's radiographic appearance have been performed.Li et al. designed a comprehensive radiological classification scheme based on callus shape and type, to monitor the progression of callus maturation during distraction osteogenesis in adolescents and adults. ${ }^{14}$ While it provided information about the nature of the callus and prediction of complications, it did not suggest an objective guide to timing for the commencement of ambulation.

Several methods of measuring and quantifying the amount of callus formed in the course of distraction osteogenesis have been explored in the past. Compared to plain radiography that requires approximately 4 weeks to visualise early regenerate bone formation, DEXA and ultrasound scans can detect regenerate bone in the distraction gap as early as 1-2 weeks following commencement of distraction. ${ }^{15}$ While ultrasound has not been shown to be useful in assessing the consolidation of regenerate bone, DEXA scans have been evaluated in both animal and clinical studies. ${ }^{16}$ Histochemical and imaging studies using DEXA scans in animal models have shown a good correlation between calcium content and mechanical strength of healing bone. ${ }^{17-19}$ It has also been used clinically to determine timing for removal of external fixators after limb lengthening by several authors. ${ }^{8,10}$ In a prospective review of 23 patients undergoing 26 limb lengthening procedures with external fixators in the femur and tibia, Maffulli et al. determined that the bone mineral content (BMC) of the regenerate bone was $67 \%$ of the preoperative BMC values at the time of removal of the external fixator. ${ }^{20}$ They also noted that the BMC values continued to increase following fixator removal, approaching normal values almost 2 years after fixator removal. Most of this increase occurred within the first 200 days following fixator removal. They found no significant differences in values between the femur and the tibia. Although DEXA scans have shown promise as an objective measuring tool, they have several disadvantages that prevent routine clinical use: Expense, limited availability and an inability to measure bony alignment.

Quantitative computed tomography scans are another modality that has been used in experimental and clinical studies as in indirect means of quantification of BMC. ${ }^{21}$ It provides high-resolution images and quantitative analysis of the mineral 
content of regenerate bone. However, like DEXA scans, it is an expensive investigative tool that is not universally available, which limits its use in clinical practice.

An ideal objective marker of regenerate bone healing must be reliable, available, safe and efficient. The PVR can be measured from the normal orthogonal digital radiographs obtained for each patient in clinic. It can be calculated while the patient is waiting in clinic and it has been shown to have a good correlation with DEXA scans in determining the BMD of regenerate bone. In a retrospective review of 40 tibial segments lengthened in 23 patients using the llizarov frame, Song et al. found a good positive correlation between bone mineral density ratio (BMDR) obtained by DEXA scanning and PVR. ${ }^{10}$ They are based on their determination of optimal time for the removal of external fixators on an equation dependent on the BMDR and PVR. They suggested the use of the PVR as an adjunct to BMDR measurements in determining optimal time for fixator removal. Zhao et al. retrospectively reviewed 17 patients who underwent bilateral tibial lengthening over a nail using an llizarov fixator. ${ }^{22}$ In this series, they determined that it was safe to commence partial weight-bearing when the PVR was one in two cortices and FWB without crutches when the PVR was one in three cortices. The lateral cortex was the quickest to attain a PVR of 1, achieving this in 28 weeks. This was followed by the posterior, medial and anterior cortices in that order. In our series, we found that the medial cortex had the closest value to 1 at the time of commencement of FWB suggesting that healing was faster on the medial side (Table 1). The medial side of the femur is the least injured during the surgical approach to the osteotomy and has abundant soft tissue coverage.

Pixel value ratio measurements from digital radiographs have been previously used to measure the progression of callus during distraction osteogenesis with intramedullary lengthening nails. ${ }^{23}$ Vulcano et al. utilising pixel density ratio (PDR) measurements determined that bone healing was achieved at a mean PDR of $0.9 .{ }^{24}$ They determined that this value was attained on the average, after 8.5 weeks of consolidation. In our study, the mean PVR at the time of clinically and radiologically determined full weightbearing was 0.94 . This is comparable to the findings by Vulcano et al. despite differences in calculation methods. They only utilised the pixel density of the distal cortex adjacent to the regenerate in their determination of the pixel density ratio. We utilised the mean pixel value of the adjacent proximal and distal cortices in determining the PVR. We felt that measuring both areas would give a more accurate evaluation of the regenerate healing. This raises an interesting question. In determining the PVR, does utilisation of only the distal cortex correlate well with values derived from the mean of the proximal and distal cortices? This will certainly be useful in situations where there is significant soft tissue overlap.

In addition to the calculation methods, our study differed from Vulcano et al. with regards to the reference point for FWB. We determined that FWB was possible at 125 days (approximately 18 weeks) following the commencement of distraction, whereas Vulcano et al. achieved FWB 8.5 weeks following the completion of distraction. It is therefore difficult to compare these two studies based on the duration of regenerate bone healing times since different time points were used as reference. We chose to use the time from the commencement of distraction because we felt that the healing process commences immediately following the osteotomy and continues through the distraction phase to the end of the consolidation phase.
There are several limitations to this study. There were no recorded cases of hardware failure or fractures in this series of patients. While this is good from the patient perspective, it does not allow us to determine if similar outcomes would have been possible if weight-bearing had commenced earlier and with a smaller PVR. In other words, the PVR we found may be overly conservative, but it seems to ensure a positive patient result. There may be a threshold where a smaller PVR value would allow a safe return to FWB sooner but we were not able to calculate it. Similarly, because the consolidation radiographs were obtained on a monthly rather than weekly basis, there was an increased gap of time between measurements. This affected how precise the PVR recommendations could be. It may be that the true threshold for safe weight-bearing is a slightly smaller PVR value than we are reporting. We also did not carry out inter- and intraobserver studies as Zhao et al. had previously demonstrated a good correlation with PVR measurements. ${ }^{22} \mathrm{~A}$ final limitation of this procedure is the effect of soft tissue overlap in the region of interest on the radiographs. We were careful to try to eliminate any measurements where there was soft tissue overlap but this occurrence has the potential to alter the pixel density and eventually the PVR measurement. ${ }^{24}$

In summary, the pixel value assesses the density of bone in pixels. It can be used to assess healing of regenerate bone by comparing its density to that of adjacent bone. As the density of the regenerate bone improves with healing, its pixel value approaches that of the normal adjacent bone. At the time of FWB in this study, three out of the four cortices had attained a PVR of at least 0.93 . We propose that this can be used as a guide to the commencement of FWB in patients undergoing intramedullary lengthening. Measurement of the pixel densities using the "ROI" tool in PACS from two cortices of a single digital X-ray is a rapid process which can be accomplished in just a few minutes. Perhaps, incorporating the formula for calculating the PVR from pixel density measurements into one of the current smartphone applications available for deformity analysis can further simplify the process. We believe that the PVR can be a practical option during routine follow-up visits, for an objective and reproducible method of determining the extent of regenerate bone healing and when it is safe to commence FWB.

\section{References}

1. Birch JG. A brief history of limb lengthening. J Pediatr Orthop 2017;37:S1-S8. DOI: 10.1097/BPO.0000000000001021.

2. Ilizarov GA. Clinical application of the tension-stress effect for limb lengthening. Clin Orthop Relat Res 1990;250(250):8-26. DOI: 10.1097/00003086-199001000-00003.

3. lobst CA. Intramedullary limb-lengthening lessons learned. JBJS Rev 2019;7(12):e2. DOI: 10.2106/JBJS.RVW.19.00034.

4. Kirane YM, Fragomen AT, Rozbruch SR. Precision of the PRECICE ${ }^{\circledR}$ internal bone lengthening nail. Clin Orthop Relat Res 2014;472(12):3869-3878. DOI: 10.1007/s11999-014-3575-0.

5. Paley D, Harris M, Debiparshad K, et al. Limb lengthening by implantable limb lengthening devices. Tech Orthop 2014;29(2):72-85. DOI: 10.1097/BTO.0000000000000072.

6. Anand A, Feldman DS, Patel RJ, et al. Interobserver and intraobserver reliability of radiographic evidence of bone healing at osteotomy sites. J Pediatr Orthop Part B 2006;15(4):271-272. DOI: 10.1097/01202412200607000-00007.

7. Den Boer FC, Bramer JAM, Patka P, et al. Quantification of fracture healing with three-dimensional computed tomography. 
Arch Orthop Trauma Surg 1998;117(6-7):345-350. DOI: 10.1007/ s004020050263.

8. Saran N, Hamdy RC. DEXA as a predictor of fixator removal in distraction osteogenesis. Clin Orthop Relat Res 2008;466(12): 2955-2961. DOI: 10.1007/s11999-008-0514-y.

9. Hazra S, Song HR, Biswal S, et al. Quantitative assessment of mineralization in distraction osteogenesis. Skeletal Radiol 2008;37(9):843-847. DOI: 10.1007/s00256-008-0495-7.

10. Song SH, Agashe M, Kim TY, et al. Serial bone mineral density ratio measurement for fixator removal in tibia distraction osteogenesis and need of a supportive method using the pixel value ratio. J Pediatr Orthop Part B 2012;21(2):137-145. DOI: 10.1097/ BPB.0b013e32834f04f3.

11. Fischgrund J, Paley D, Suter C. Variables affecting time to bone healing during limb lengthening. Clin Orthop Relat Res 1994;301(301):31-37. DOI: 10.1097/00003086-199404000-00006.

12. Skaggs DL, Leet Al, Money MD, et al. Secondary fractures associated with external fixation in pediatric femur fractures. J Pediatr Orthop 1999;19(5):582-586. DOI: 10.1097/01241398-19990900000005.

13. Starr KA, Fillman R, Raney EM. Reliability of radiographic assessment of distraction osteogenesis site. J Pediatr Orthop 2004;24(1):26-29. DOI: 10.1097/01241398-200401000-00006.

14. Li R, Saleh M, Yang L, et al. Radiographic classification of osteogenesis during bone distraction. J Orthop Res 2006;24(3):339-347. DOI: 10.1002/jor.20026.

15. Eyres KS, Bell MJ, Kanis JA. Methods of assessing new bone formation during limb lengthening. ultrasonography, dual energy $x$-ray absorptiometry and radiography compared. J Bone Jt Surg - Ser B 1993;75-B(3):358-364. DOI: 10.1302/0301-620X.75B3. 8496200.
16. Babatunde OM, Fragomen AT, Rozbruch SR. Noninvasive quantitative assessment of bone healing after distraction osteogenesis. HSS J 2010;6(1):71-78. DOI: 10.1007/s11420-009-9130-y.

17. Powell ES, Lawford PV, Duckworth T, et al. Is callus calcium content an indicator of the mechanical strength of healing fractures? An experimental study in rat metatarsals. J Biomed Eng 1989;11(4):277281. DOI: 10.1016/0141-5425(89)90059-9.

18. Reichel H, Lebek S, Alter $\mathrm{C}$, et al. Biomechanical and densitometric bone properties after callus distraction in sheep. Clin Orthop Relat Res 1998;357:237-246. DOI: 10.1097/00003086-199812000-00030.

19. Chakkalakal DA, Lippiello L, Wilson RF, et al. Mineral and matrix contributions to rigidity in fracture healing. J Biomech 1990;23(5):425-434. DOI: 10.1016/0021-9290(90)90297-G.

20. Maffulli N, Cheng JCY, Sher A, et al. Bone mineralization at the callotasis site after completion of lengthening. Bone 1999;25(3): 333-338. DOI: 10.1016/S8756-3282(99)00168-4.

21. Aronson J, Shin HD. Imaging techniques for bone regenerate analysis during distraction osteogenesis. J Pediatr Orthop 2003;23(4):550-560. DOI: 10.1097/01241398-200307000-00025.

22. Zhao L, Fan Q, Venkatesh $\mathrm{KP}$, et al. Objective guidelines for removing an external fixator after tibial lengthening using pixel value ratio: a pilot study. Clin Orthop Relat Res 2009;467(12):3321-3326. DOI: 10.1007/s11999-009-1011-7.

23. Sun XT, Easwar TR, Stephen $M$, et al. Comparative study of callus progression in limb lengthening with or without intramedullary nail with reference to the pixel value ratio and the ruli's classification. Arch Orthop Trauma Surg 2011;131(10):1333-1340. DOI: 10.1007/s00402011-1302-9.

24. Vulcano E, Markowitz JS, Ali S, et al. Assessment of bone healing during antegrade intramedullary rod femur lengthening using radiographic pixel density. J Am Acad Orthop Surg 2018;26(18): e388-e394. DOI: 10.5435/JAAOS-D-16-00949. 